\title{
Acute exposure to an electric field induces changes in human plasma lysophosphatidylcholine (lysoPC)-22:4 levels: Molecular insight into the docking of lysoPC-22:4 interaction with TRPV2
}

\author{
Nakagawa-Yagi $\mathrm{Y}^{1 *}$, Hara $\mathrm{H}^{1}$, Nakanishi $\mathrm{H}^{2}$, Tasaka $\mathrm{T}^{3}$ and Hara $\mathrm{A}^{1}$ \\ ${ }^{1}$ Hakuju Institute for Health Science Co., Ltd., 37-5 Tomigaya 1-chome, Shibuya-ku, Tokyo, Japan \\ ${ }^{2}$ Akita Lipid Technologies LLC., 1-2 Nukazuka, Yanagida, Akita, Akita, Japan \\ ${ }^{3}$ Affinity Science Corporation, 1-1-1 Nishigotanda, Shinagawa-ku, Tokyo, Japan
}

\begin{abstract}
Medical treatment using high-voltage electric potential (HELP) devices to generate an electric field (EF) is an alternative therapy commonly used in Japan. However, the underlying mechanisms of the potential health benefits are not fully understood. To address this issue, we investigated the levels of lyso-form phospholipids using selected reaction monitoring (SRM) analysis in plasma samples obtained from healthy human subjects before and after a single HELP exposure (9 $\mathrm{kV} / \mathrm{electrode}+$ $9 \mathrm{kV} /$ electrode, $30 \mathrm{~min}$ ). Lysophosphatidylcholine (lysoPC)-22:4 was significantly upregulated after HELP exposure. However, there was no effect on the levels of lysophosphatidic acid (lysoPA), or other lysoPC species. LysoPC is known to accelerate intestinal movement as a putative endogenous activator of transient receptor potential vanilloid 2 (TRPV2). We further examined the in silico docking simulation of lysoPC-22:4 with TRPV2. Docking results showed that lysoPC-22:4 has good binding energy $(-8.2 \mathrm{kcal} / \mathrm{mol})$. Our findings provide new insight into the molecular mechanisms of constipation alleviation by EF therapy.
\end{abstract}

\begin{abstract}
Abbreviations
EF: electric field; FA: fatty acid; GPR119: G protein-coupled receptor 119; HELP: high-voltage electric potential; lysoPA: lysophosphatidic acid; lysoPC: lysophosphatidylcholine; lysoPC-22:4 : (2-\{[(2R)-3[(7Z,10Z,13Z,16Z)-docosa-7,10,13,16-tetraenoyloxy]-2-hydropropyl phosphonato]oxy\}ethyl)trimethylazanium; PA: phosphatidic acid; PC: phosphatidylcholine; RTx: resiniferatoxin; SRM: selected reaction monitoring; TRPM8: transient receptor potential melastatin 8; TRPV1: transient receptor potential vanilloid 1; and TRPV2: transient receptor potential vanilloid 2
\end{abstract}

\section{Introduction}

A therapeutic device to expose the human body to high-voltage electric potential (HELP) has been approved by the Ministry of Health, Labour and Welfare in Japan [1-15]. High-voltage electric field (EF) therapy is reported to be an effective treatment for stiff shoulders, headache, insomnia, and chronic constipation [1-15]. In the case of constipation, considerable evidence of efficacy has been obtained from several clinical studies of functional constipation and chronic constipation [16-17]. Taken together, the results of these studies suggest that HELP exposure may present an alternative therapy for several conditions, although the mechanisms of action remain elusive. Our previous attempts to find a HELP exposure-induced biomarker using plasma metabolomics have led to the detection of lipid-derived signaling molecules such as oleoylethanolamide (OEA), cis-8,11,14eicosatrienoic acid, 9-hydroxyoctadecadienoic acid (9-HODE), 13HODE, 13-hydroperoxyoctadecadienoic acid (13-HpODE), and 3-hydroxybutyrate [18-21]. Endogenous lipid-derived signaling molecules have been suggested as candidate molecules that represent the interface between symptoms and electroceutical target proteins [18-21]. Currently, more recent methods for the quantification of lipidderived signaling molecules have taken advantage of SRM analysis [2226]. Therefore, we hypothesized that increased plasma fatty acid levels after EF exposure may be linked to changes in lyso-form phospholipids in plasma. A recent study by Mihara et al. reported that gastrointestinal transit in vivo is accelerated by the lyso-form phospholipid lysoPC [27]. The observations of Mihara et al. led us to study changes in lysoPC, using SRM analysis on plasma samples obtained from healthy subjects before and after exposure to a single HELP stimulation [27]. Here we report that lysoPC-22:4 can be upregulated by HELP exposure ( 9 $\mathrm{kV} /$ electrode $+9 \mathrm{kV} /$ electrode, $30 \mathrm{~min}$ ). Because lysoPC treatment of isolated myenteric neurons induces transient receptor potential vanilloid 2 (TRPV2)-mediated responses [27], we carried out a binding study using AutoDock Vina docking software to explore the interactions of lysoPC-22:4 with the active site of TRPV2.

Correspondence to: Yuzo Nakagawa-Yagi, Ph.D, Hakuju Institute for Health Science Co., Ltd., 37-5 Tomigaya 1-chome, Shibuya-ku, Tokyo, Japan, E-mail: yagi@hakuju.co.jp

Key words: lysophosphatidylcholine, lysoPC-22:4, TRPV2, lipidomics, electric field therapy

Received: March 07, 2017; Accepted: March 15, 2017; Published: March 18, 2017 


\section{Materials and methods}

\section{EF exposure}

The system used for EF exposure has been previously described [1821]. The EF system was equipped with a transformer, a seat, and two insulator-covered electrodes. One electrode was placed on a floor plate on which the subject's feet were located, and the other was placed above the subject's head. EF generated by the HELP apparatus (Healthtron PRO-18T; Hakuju Institute for Health Science Co., Ltd., Tokyo, Japan) was uniformly created by transforming a $50 \mathrm{~Hz}$ alternating current at $18 \mathrm{kV}$ ( $9 \mathrm{kV} /$ electrode $+9 \mathrm{kV} /$ electrode). The safety of this system for human use was established by the Japanese government in 1963.

\section{Subjects}

Fifty healthy adults [ 21 males and 29 females; mean age, $46.5 \pm 0.9$ years; mean body mass index (BMI), $21.9 \pm 0.4 \mathrm{~kg} / \mathrm{m}^{2}$ ] participated in experiment 1 (exposure condition: $9 \mathrm{kV} /$ electrode $+9 \mathrm{kV} /$ electrode, 30 min). Twenty-five healthy adults ( 9 males and 16 females; mean age, $46.1 \pm 1.1$ years; BMI, $\left.22.2 \pm 0.5 \mathrm{~kg} / \mathrm{m}^{2}\right)$ participated in experiment 2 (exposure condition: $9 \mathrm{kV} /$ electrode $+9 \mathrm{kV} /$ electrode, $30 \mathrm{~min}$ ). All experiments were performed in the morning and all participants signed an informed consent form after receiving verbal and written information about the study. All experiments were conducted in accordance with the Declaration of Helsinki and the study protocol was approved by the human ethics committee of Hakuju Institute for Health Science Co., Ltd. (Tokyo, Japan).

\section{Plasma preparation}

Blood samples were collected in vacutainer tubes coated with ethylenediaminetetraacetic acid (VP-NA070K; Terumo Corporation, Tokyo, Japan) and immediately centrifuged at $800 \times \mathrm{g}$ for $5 \mathrm{~min}$ to separate plasma from other cellular materials. Plasma was then transferred to a fresh Eppendorf tube and stored at $-80^{\circ} \mathrm{C}$ until processing.

\section{Phospholipid preparation}

Comprehensive analysis of phospholipids was conducted in essentially the same manner as described previously [28-29]. Briefly, total phospholipids were extracted from the plasma with the Bligh-Dyer method [30]. Aliquots of the lower/organic phase were evaporated to dryness under $\mathrm{N}_{2}$, and the residue was dissolved in methanol for LC-MS/MS measurements of phosphatidylcholine (PC). To analyze phosphatidic acid (PA), another aliquot of the same lipid extract was added to an equal volume of methanol before being loaded onto a DEAE cellulose column (Santa Cruz Biotechnology, Dallas, TX, USA) pre-equilibrated with chloroform. After successive washes with chloroform/methanol $(1: 1, \mathrm{v} / \mathrm{v})$, the acidic phospholipids were eluted with chloroform/methanol/HCl/water (12:12:1:1, v/v), followed by evaporation to dryness to give a residue, which was again dissolved in methanol. The resultant fraction was subjected to a methylation reaction with TMS-diazomethane before LC-MS/MS analysis [31].

\section{Mass spectrometric analyzes}

LC-electrospray ionization-MS/MS analysis was performed with an UltiMate 3000 LC system (Thermo Fisher Scientific, Waltham, MA, USA) equipped with HTC PAL autosampler (CTC Analytics AG, Lake Elmo, MN, USA). A $10 \mu \mathrm{L}$ aliquot of the lipid samples was injected and the lipids were separated on a Waters X-Bridge C18 $(3.5 \mu \mathrm{m}, 150$ $\mathrm{mm} \times 1.0 \mathrm{~mm}$ i.d., Waters Corporation, Milford, MA, USA) at room temperature $\left(25^{\circ} \mathrm{C}\right)$ using a gradient solvent system as follows: mobile phase A [isopropanol/methanol/water (5:1:4, v/v/v) supplemented with $5 \mathrm{mM}$ ammonium formate and $0.05 \%$ ammonium hydroxide]; mobile phase B (isopropanol supplemeted with $5 \mathrm{mM}$ ammpnium formate and $0.05 \%$ ammonium hydroxide) ratios of 70\%/30\% (0 min), $50 \% / 50 \%$ ( $2 \mathrm{~min}$ ), $20 \% / 80 \%$ (13 $\mathrm{min}$ ), $5 \% / 95 \%$ ( $15-30 \mathrm{~min}$ ), $95 \% / 5 \%$ $(31-35 \mathrm{~min})$, and $70 \% / 30 \%(35-45 \mathrm{~min})$. Flow rate was $20 \mu \mathrm{L} / \mathrm{min}$. Phospholipid species were measured by selected reaction monitoring (SRM) in positive ion mode with a TSQ Vantage AM mass spectrometer (Thermo Fisher Scientific, Waltham, MA, USA). The characteristic fragments of individual phospholipids were detected by the product ion scan (MS/MS mode). Chromatographic peak areas were used for comparative quantitation of each molecular species (e.g., 38:6, 40:6) in a given class of the phospholipids (e.g., PA, PC). The specific detection of individual lipids was performed using multiple reaction monitoring. Multiple reaction monitoring $(\mathrm{MRM}) \mathrm{m} / \mathrm{z}$ transitions were lysoPC-22:4 $=572.4 / 184.1$.

\section{Molecular modeling and docking study}

The structure of the full-length TRPV2 channel (5HI9; Protein Data Bank Japan) was used for the molecular docking study. The docking study of the binding of lysoPC-22:4 to TRPV2 was performed using AutoDock Vina docking software (Dr. Oleg Trott, The Scripps Research Institute, CA, USA) [32]. The docking experiment was performed five times and yielded 100 candidate conformations.

\section{Statistical analysis}

Data were analyzed using Welch's $t$-test. A probability $(p)$ value $<$ 0.05 was considered statistically significant.

\section{Results}

\section{Effect of HELP exposure on lysoPC and PC in plasma from healthy humans}

We assessed lysoPC in the plasma obtained from 50 healthy participants using SRM analysis (Figure 1 and Figure 2). HELP exposure $(9 \mathrm{kV} /$ electrode $+9 \mathrm{kV} /$ electrode, $30 \mathrm{~min})$ resulted in significantly higher plasma levels of lysoPC-22:4 than pre-exposure levels $(1.47$-fold; $p=0.027)$. Under these conditions, HELP exposure did not affect the levels of lysoPC-14:0, lysoPC-16:0, lysoPC-16:1, lysoPC-18:0, lysoPC-18:1, lysoPC-18:2, lysoPC-20:0, lysoPC-20:1, lysoPC-20:2, lysoPC-20:3, lysoPC-20:4, lysoPC-20:5, lysoPC-22:5, or lysoPC-22:6 (Figure 2).

We assessed $\mathrm{PC}$ in the plasma obtained from 50 healthy participants using SRM analysis. HELP exposure $(9 \mathrm{kV} /$ electrode $+9 \mathrm{kV} /$ electrode, $30 \mathrm{~min}$ ) did not affect the levels of PC-32:0, PC-34:0, PC-34:1, PC-34:2, PC-36:0, PC-36:1, PC-36:2, PC-36:3, PC-36:4, PC-38:4, PC-38:5, PC40:4, PC:40:5, or PC-40:6 (Figure 3).

\section{Effect of HELP exposure on lysoPA and PA in plasma from healthy humans}

We assessed lysoPA in the plasma obtained from 25 healthy participants using SRM analysis (Figure 4). HELP exposure did not affect the levels of lyso-PA-16:0, lysoPA-18:0, lysoPA-18:1, lysoPA-18:2, lysoPA-20:4, or lysoPA-22:6 (Figure 4).

We assessed PA in the plasma obtained from 25 healthy participants using SRM analysis. HELP exposure $(9 \mathrm{kV} /$ electrode $+9 \mathrm{kV} /$ electrode, $30 \mathrm{~min}$ ) did not affect the levels of PA-32:0, PA-32:1, PA-34:0, PA-34:1, PA-34:2, PA-36:0, PA-36:1, PA-36:2, PA-36:3, PA-36:4, PA-38:3, PA38:4, PA-38:5, PA-38:6, PA-40:4, PA-40:5, or PA-40:6 (Figure 5). 


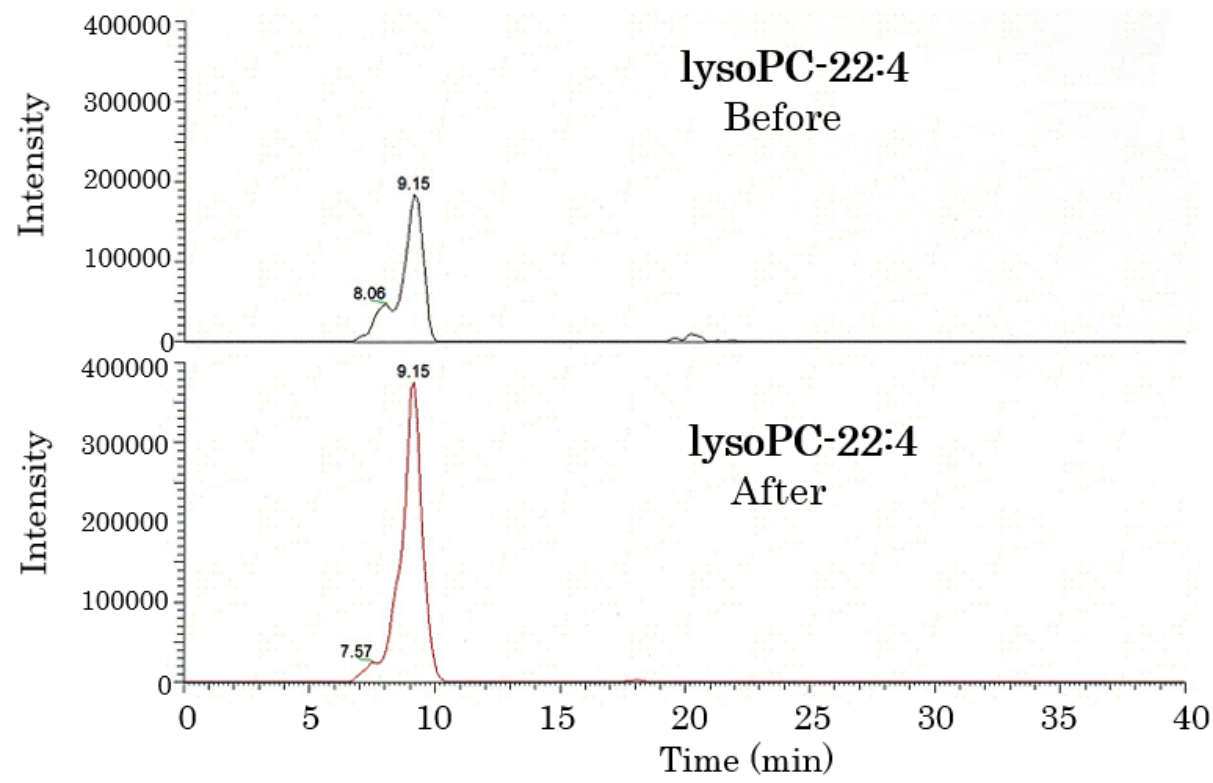

Figure 1. Lipidomic analysis of the plasma of healthy humans before and after HELP exposure for $30 \mathrm{~min}$. Typical lysoPC-22:4 peak in the plasma of healthy humans. LysoPC-22:4 was detected by SRM analysis.

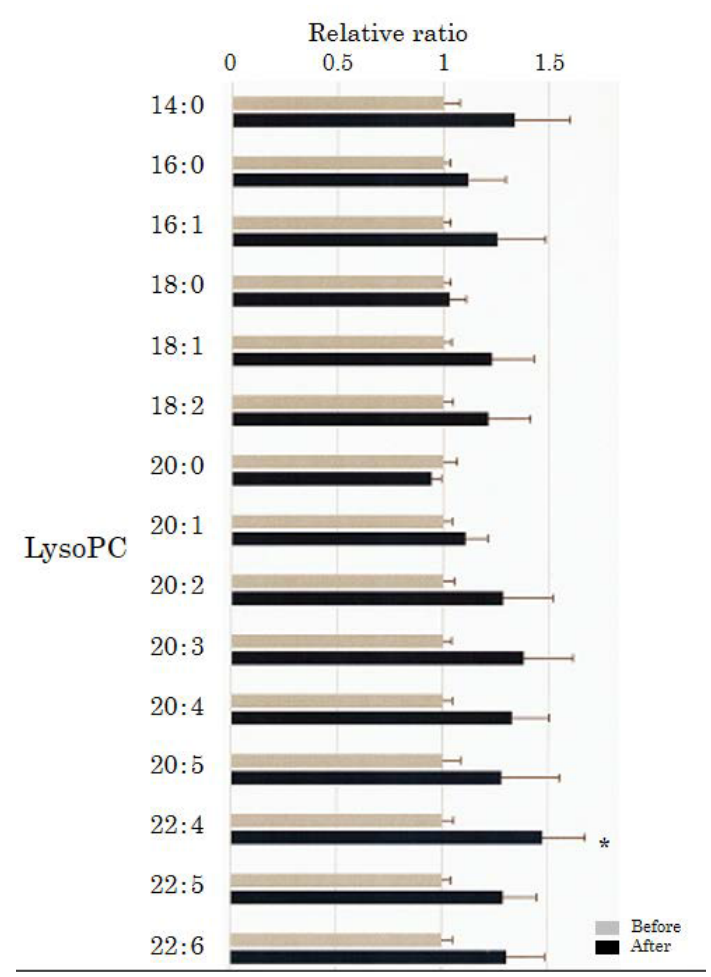

Figure 2. Effect of HELP exposure $(9 \mathrm{kV} /$ electrode $+9 \mathrm{kV} / \mathrm{electrode}, 30 \mathrm{~min})$ on lysoPC in the plasma of healthy humans. Relative ratio (after/before) of lysoPC in plasma before and after EF exposure. Results are presented as mean $\pm \operatorname{SEM}(\mathrm{n}=50) . * p<0.05$ compared with before.

\section{Docking of lysoPC-22:4 on TRPV2}

LysoPC is known to induce gastrointestinal tract transit as an activator of TRPV2 [27]. Therefore, we hypothesized that increased plasma lysoPC-22:4 levels after HELP exposure may be linked to

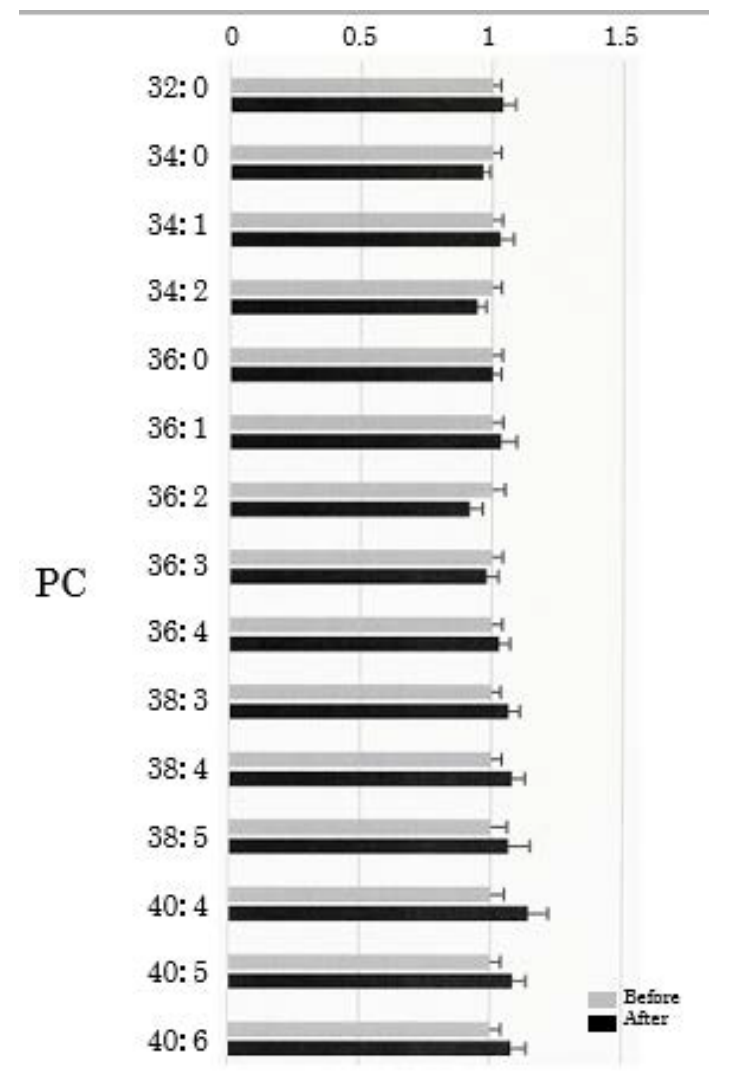

Figure 3: Effect of HELP exposure $(9 \mathrm{kV} /$ electrode $+9 \mathrm{kV} /$ electrode, $30 \mathrm{~min})$ on PC in the plasma of healthy humans. Relative ratio (after/before) of PC in plasma before and after EF exposure. Results are presented as mean $\pm \operatorname{SEM}(n=50)$.

alleviation of constipation in humans. We examined the in silico docking of lysoPC-22:4 in the active site of TRPV2 using AutoDock Vina software. We set the number of output poses to 20, with a total of 100 candidate conformations. Cluster analysis was performed using 

the docking of lysoPC-22:4 interaction with TRPV2

KNIME [33], and classified results into 32 conformation groups. Table 1 shows the obtained docking score and cluster ID. As shown in Table 1 , the conformation group of the cluster ID-31 was the largest. Next, eight conformations of cluster ID-31 with binding energies lower than $-7.85 \mathrm{kcal} / \mathrm{mol}$ were compared. As shown in Figure $6 \mathrm{a}$, similar binding poses were observed. In those poses, cluster ID-31 was the best conformation (seed: 101 , mode 1) with lowest binding energy -8.2 $\mathrm{kcal} / \mathrm{mol}$ (Table 1, Figure 6b). LysoPC-22:4 formed hydrogen bonds with Ser526, Gln530, and Asn639 (Figure 6c). The results indicated that lysoPC-22:4 would bind to the TRPV2 channel.

\section{Discussion}

In this study, we showed that lysoPC-22:4 in healthy human subjects is sensitive to acute EF exposure. Notably, the absence of lysoPA

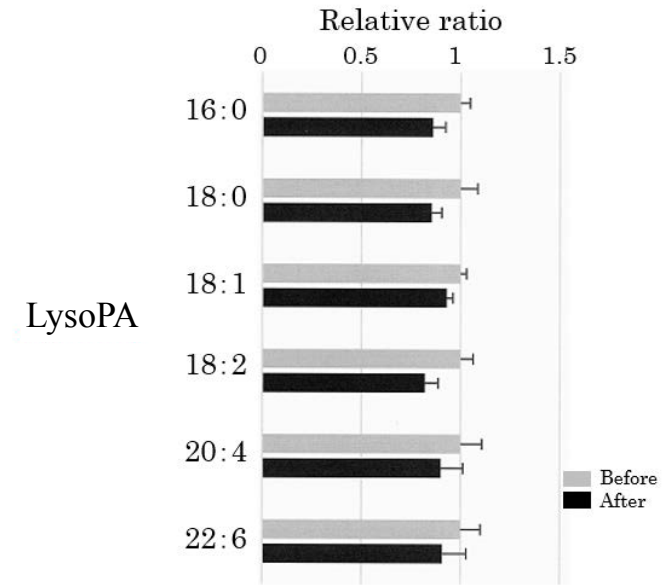

Figure 4: Effect of HELP exposure ( $9 \mathrm{kV} / \mathrm{electrode}+9 \mathrm{kV} / \mathrm{electrode}, 30 \mathrm{~min})$ on lysoPA in the plasma of healthy humans. Relative ratio (after/before) of lysoPA in plasma before and after EF exposure. Results are presented as mean \pm SEM $(n=25)$.

Table 1. Docking score and cluster ID. response indicates that the response to lysoPC-22:4 is not by an adverse nonspecific action on membrane lipids. The molecular mechanisms of

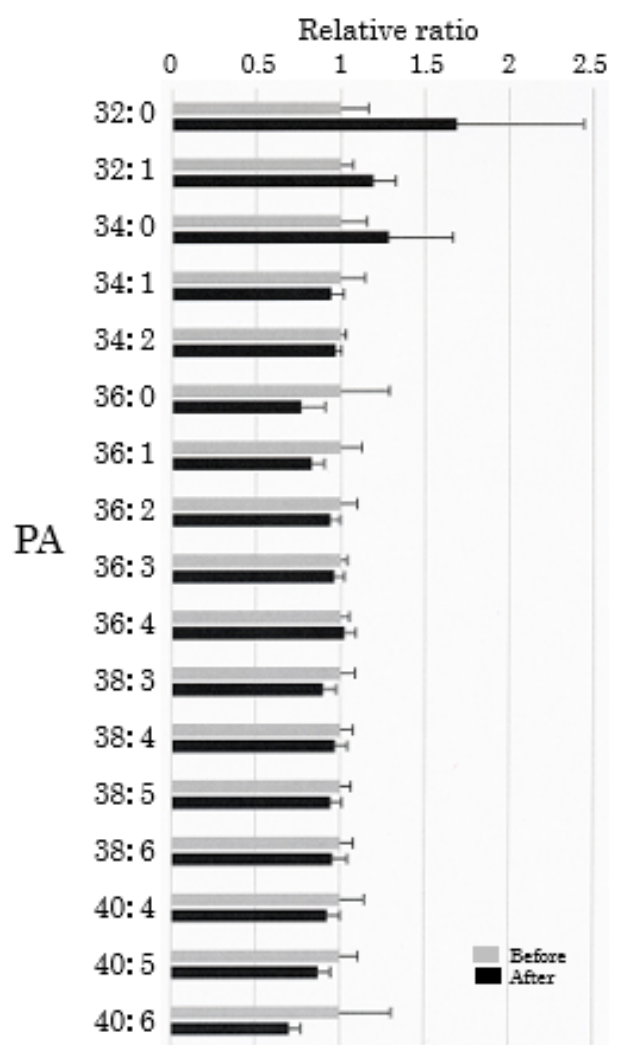

Figure 5: Effect of HELP exposure ( $9 \mathrm{kV} /$ electrode $+9 \mathrm{kV} /$ electrode, $30 \mathrm{~min})$ on PA in the plasma of healthy humans. Relative ratio (after/before) of PA in plasma before and after EF exposure. Results are presented as mean $\pm \operatorname{SEM}(n=25)$.

\begin{tabular}{|c|c|c|c|c|c|c|c|c|c|c|}
\hline \multirow[t]{3}{*}{ Pocket } & \multicolumn{10}{|l|}{ Seed } \\
\hline & \multicolumn{2}{|l|}{101} & \multicolumn{2}{|l|}{102} & \multicolumn{2}{|l|}{103} & \multicolumn{2}{|l|}{104} & \multicolumn{2}{|l|}{105} \\
\hline & $\begin{array}{l}\text { Affinity } \\
\text { kcal/mol }\end{array}$ & Cluster ID & $\begin{array}{l}\text { Affinity } \\
\text { kcal/mol }\end{array}$ & Cluster ID & $\begin{array}{l}\text { Affinity } \\
\text { kcal/mol }\end{array}$ & Cluster ID & $\begin{array}{l}\text { Affinity } \\
\text { kcal/mol }\end{array}$ & Cluster ID & $\begin{array}{l}\text { Affinity } \\
\text { kcal/mol }\end{array}$ & Cluster ID \\
\hline Mode 2 & -8.1 & 32 & -7.9 & 31 & -8.0 & 28 & -7.5 & 29 & -8.0 & 26 \\
\hline Mode 3 & -8.1 & 30 & -7.8 & 31 & -7.9 & 31 & -7.4 & 31 & -7.9 & 31 \\
\hline Mode 4 & -8.0 & 28 & -7.8 & 5 & -7.8 & 7 & -7.4 & 32 & -7.9 & 27 \\
\hline Mode 5 & -7.9 & 31 & -7.7 & 31 & -7.8 & 8 & -7.4 & 28 & -7.9 & 31 \\
\hline Mode 6 & -7.9 & 31 & -7.7 & 32 & -7.8 & 25 & -7.4 & 31 & -7.9 & 26 \\
\hline Mode 7 & -7.9 & 32 & -7.7 & 31 & -7.8 & 31 & -7.3 & 12 & -7.8 & 31 \\
\hline Mode 8 & -7.8 & 1 & -7.7 & 31 & -7.7 & 31 & -7.3 & 32 & -7.8 & 32 \\
\hline Mode 9 & -7.8 & 2 & -7.7 & 25 & -7.7 & 9 & -7.3 & 31 & -7.8 & 29 \\
\hline Mode 10 & -7.8 & 22 & -7.6 & 31 & -7.6 & 26 & -7.3 & 29 & -7.8 & 31 \\
\hline Mode 12 & -7.7 & 32 & -7.6 & 23 & -7.6 & 10 & -7.2 & 29 & -7.8 & 15 \\
\hline Mode 13 & -7.7 & 20 & -7.6 & 28 & -7.6 & 32 & -7.2 & 30 & -7.8 & 31 \\
\hline Mode 14 & -7.7 & 3 & -7.6 & 32 & -7.5 & 25 & -7.2 & 32 & -7.8 & 31 \\
\hline Mode 15 & -7.7 & 20 & -7.6 & 23 & -7.5 & 27 & -7.2 & 31 & -7.7 & 29 \\
\hline Mode 16 & -7.7 & 25 & -7.5 & 32 & -7.5 & 11 & -7.2 & 32 & -7.7 & 27 \\
\hline Mode 17 & -7.7 & 4 & -7.5 & 19 & -7.5 & 32 & -7.2 & 27 & -7.7 & 16 \\
\hline Mode 18 & -7.7 & 19 & -7.5 & 30 & -7.5 & 26 & -7.1 & 21 & -7.6 & 17 \\
\hline Mode 19 & -7.7 & 32 & -7.5 & 22 & -7.4 & 28 & -7.1 & 24 & -7.6 & 20 \\
\hline Mode 20 & -7.5 & 31 & -7.4 & 6 & -7.4 & 27 & -7.0 & 14 & -7.5 & 18 \\
\hline
\end{tabular}


a

b

C

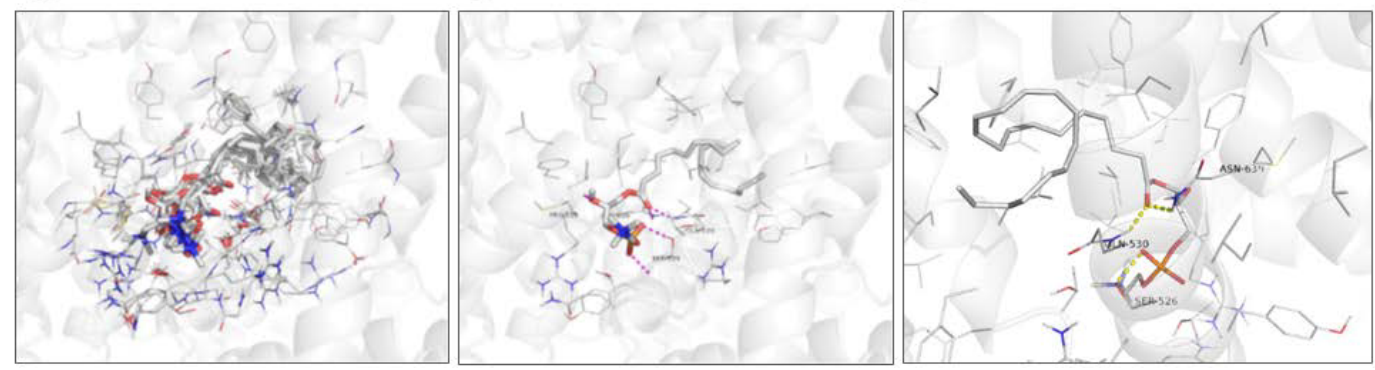

Figure 6: View of the conformation of lysoPC-22:4 docked in the TRPV2 channel. (a) Eight conformations of cluster ID-31 with binding energies lower than - 7.85 kcal/mol. (b) Best conformation of lysoPC-22:4 (seed: 101, mode 1) with lowest binding energy ( $-8.2 \mathrm{kcal} / \mathrm{mol})$. (c) Binding mode of lysoPC-22:4 in TRPV2 channel.

changes in lysoPC-22:4 levels following EF exposure are complex and can be interpreted in several ways. Interestingly, Thuren et al. reported that phospholipase $\mathrm{A}_{2}\left(\mathrm{PLA}_{2}\right)$-catalyzed hydrolysis is elevated by EF [34]. PLA 2 enzymes hydrolyze the ester of glycerophospholipids to release lysophospholipids and a free unsaturated fatty acid [35]. In plasma metabolomics, we have previously documented EF exposure (9 $\mathrm{kV} /$ electrode $+9 \mathrm{kV} /$ electrode)-induced increases of approximately 1.52-fold, 1.47-fold, 1.41-fold, 1.46-fold, 1.46-fold, and 1.51-fold for oleic acid, linoleic acid, arachidonic acid (FA-20:4), FA-22:4, FA-22:5, and cis-4,7,10,13,16,19-docosahexaenoic acid (FA-22:6), respectively [18]. The results of the present study showed that EF exposure induced a 1.47-fold increase in human plasma lysoPC-22:4 levels. Thus, it is reasonable to speculate that $\mathrm{EF}$ exposure upregulates lysoPC-22:4 through the activation of PLA ${ }_{2}$. Further studies are needed to identify the lysoPC-22:4 signaling pathways induced by EF exposure.

Another goal of the present study was to gain insight into the molecular mechanisms of constipation alleviation by EF therapy. Interestingly, Mihara et al. reported that intragastric administration of TRPV2 agonists such as probenecid and lysoPC induced an enhancement of gastrointestinal transit in vivo through TRPV2 activation in mice [22]. Unfortunately, there is no commercially available lysoPC-22:4 as a pure chemical reagent for pharmacological experiments. Thus, it will take some time for us to investigate the effects of lysoPC-22:4 on gastrointestinal tract transit in vivo. An increasing number of reports on virtual simulation have appeared in the literature [18-19, 36-37]. In silico molecular docking studies have been used to support their pharmacological results. To date, research regarding the effect of endogenous ligand on TRPV2 channels is scarce [3839]. Most published studies were carried out using a TRPV1 model [40-43]. Prescott et al. reported activation of the TRPV1 channel by phosphatidylinositol 4,5-biphosphate (PIP 2 ) [44], while Nieto-Posadas et al. reported lysoPA-stimulated TRPV1 channel activation through a C-terminal binding site [45]. TRPV1 shares about $50 \%$ sequence identity with TRPV2 [46]. Huynh et al. recently reported the structure of the full-length rat TRPV2 channel by cryo-electron microscopy [38], while Zubcevic et al. reported the atomic model of rabbit TRPV2 [39]. In crystallographic analyzes of human TRPV2, there is limited information on the structure of the ankyrin repeat domain of TRPV2. In the present study, the docking analysis showed that lysoPC-22:4 has good binding affinity $(-8.2 \mathrm{kcal} / \mathrm{mol})$. Moreover, it interacted with Ser526, Gln530, and Asn639. Interestingly, a previous study of rat TRPV2 channels with resiniferatoxin (RTx) binding pockets reported hydrogen bonding to Gln530 [47]. Further studies are needed to identify a putative lysoPC-22:4 binding pocket in human TRPV2. Considerable evidence for the health benefits of EF treatment have been obtained from clinical data of eighty-five patients with functional constipation [17]. Moreover, Zhang et al. reported that EF treatment for 40 days induced an approximately $60 \%$ cure rate in subjects with functional constipation [16]. On the other hand, Mihara et al. reported the involvement of TPRV2 in nitric oxide (NO)-mediated intestinal relaxation [27]. Thus, it is reasonable to speculate that EF exposure might alleviate constipation through the binding of TRPV2 by lysoPC-22:4. However, lysoPC also activates the cold-sensitive channel transient receptor potential melastatin 8 (TRPM8) and G protein-coupled receptor 119 (GPR119) [48-50], raising the possibility that these receptors also serve as targets for lysoPC-22:4 during EF exposure.

In conclusion, acute EF exposure exerted marked effects on plasma lysoPC-22:4 levels in healthy subjects, and in silico molecular docking of lysoPC-22:4 was observed for the TRPV2 channel. Our findings provide insight into the molecular mechanisms behind the health benefits induced by the HELP device, which may also be important for gastrointestinal motility.

\section{Competing interests}

Yuzo Nakagawa-Yagi, Hiroyuki Hara, Akikuni Hara are employed by Hakuju Institute for Health Science Co., Ltd., HN is employed by Akita Lipid Technologies LLC., and TT is employed by Affinity Science Corporation. All other authors have no competing interests.

\section{Authors' contributions}

Yuzo Nakagawa-Yagi designed and supervised the research, and wrote the manuscript. Hiroki Nakanishi performed SRM. Tomohiko Tasaka performed the molecular modeling. Yuzo Nakagawa-Yagi, Hiroyuki Hara, and Akikuni Hara performed the biophysical experiments. All authors have read and approved the final version of the manuscript.

\section{Acknowledgment}

We thank Dr. Makoto Kikuchi (Professor Emeritus, National Defense Medical College, Japan) for encouragement.

\section{References}

1. Ishikawa K (1929) Tuberculosis and physical therapy (in Japanese). In: J. Kawahara (ed.), Practice for clinical doctor - special issue on latest clinical tuberculosis, Daido Gakukan Press, Fukuoka, Japan, 1204-1209.

2. Hara H (1961) On the effect of AC. Electrostatic high voltage potential load upon the blood-electrolytes (in Japanese). Niigata Medical J 75: 265-273.

3. Ito F, Furuya K (1981) The effect of high voltage alternating current upon a human body the change of blood pressure, endocrine system and serum lipids (in Japanese). $J$ 
Nakagawa-Yagi Y (2017) Acute exposure to an electric field induces changes in human plasma lysophosphatidylcholine (lysoPC)-22:4 levels: Molecular insight into the docking of lysoPC-22:4 interaction with TRPV2

\section{Jpn Sci Balneol Climatol Phys Med 45: 6-17.}

4. Isaka K, Nishimura R, Arase S, Takiwaki H, Osaki K, et al. (1998) Dosimetry and exposure experiments for extremely low frequency high-tension electric field therapy. EMC '98 Rome International Symposium on Electromagnetic Comoatibility D 1: 204207.

5. Nawarat S, Iomsai K, Jantanam P, Kauengtip Y (1999) Effects of electrical Healthtron on curing of non-communicable diseases: Case study of Banlad hospital Petchaburi province (in Thai). Region 4 Medical J 18: 139-149.

6. Ito F (2000) The role of electric field therapeutic device (Healthtron) in the therapy of acute low back pain (in Japanese). J Jpn Sci BalneolClimatol Phys Med 63: 127 -137.

7. Siripanichgon K, Otrakul A, Suparp J, Sirikulchayanonta C, Charupoonphol P, et al (2000) Clinical observation of Healthtron therapy (in Thai). J Public Health (Bangkok) 30: $19-29$.

8. Sirikulchayanonta C, Siripanichgon K, Otrakul A, Suparp J, Charupoonphol P, et al. (2001) The effect of Healthtron on serum lipid levels among the middle-aged: Preliminary report. J Public Health (Bangkok) 31: 63-70.

9. Sung KK, Hwang CY, Lee SK, Lee SY, Cheong SS, et al. (2002) The clinical effect of high AC field therapy (Healthtron) on peripheral circulatory disturbance and functional outcome of rehabilitation in CVA patients (in Korean). Korean J Oriental PhysiolPathol16: 609-615.

10. Ito F, Ohsaki K, Takahashi K, Hara H (2005) The effects of electric field therapeutic device (Healthtron) on the stiffness in the neck and shoulder area - changes in subjective symptoms, blood circulation and the autonomic nervous system (in Japanese). J Jpn Sci BalneolClimatol Phys Med 68: 110-121.

11. Li Z, Nan P, Wei C, Wang QH, Li XY, et al. (2012) Effect of high-voltage electrostatic therapy on sleep disorder in older adults (in Chinese). Chin J Rehabil Theory Pract 18 (3): 286-288.

12. Shinba T, Takahashi K, Kanetaka S, Nedachi T, Yamaneki M, et al. (2012) A pilot study on electric field therapy for chronic pain with no obvious underlying diseases (in Japanese). Soc Integrative Med Jpn 5: 68-72.

13. Naito Y, Yamaguchi S, Mori Y, Nakajima K, Hashimoto S, et al. (2013) A randomized, double-blind, sham-controlled study of static electric field therapy by high voltage alternating current for active rheumatoid arthritis. J Clin Biochem Nutr 53: 63-67.

14. Yanamoto H, Nakajo Y, Kataoka H, Iihara K (2013) High voltage electric potentials to enhance brain-derived neurotrophic factor levels in the brain. Front NeurolNeurosci32: 129-138.

15. Mitani Y, Matsugi A, Okano H, Nedachi T, Hara H (2015) Effect of exposure to a high-voltage alternating current electric field on muscle extensibility.J Jpn Sci BalneolClimatol Phys Med 78: 244-252.

16. Zhang U, Li Y, Lei X, Ye Y, Liu X (2002) Clinical application of high-voltage potential treatment (in Chinese). Chinese J Clinical Rehabilitation 6: 2439

17. He SJ, Fu LT, Xu WG (2006) Clinical application of high-voltage potential treatment for neurasthenia and functional constipation (in Chinese). Chinese J Traditional Western Med 7: 39.

18. Nakagawa-Yagi Y, Hara H, Fujimori T, Yamaguchi T, Midorikawa A, et al. (2014) Non-targeted human plasma metabolomics reveals the changes in oleoylethanolamide, a lipid-derived signaling molecule, by acute exposure of electric field. IntegrMol Med 1: 29-37.

19. Nakagawa-Yagi Y, Hara H, Yoshida Y, Midorikawa A, Hara A, et al. (2015) Discovery of a novel effect of electric field exposure on human plasma beta-endorphin and interleukin-12 levels: Insight into mechanisms of pain alleviation and defense against infection by electric field therapy. Integr Mol Med 2: 200-204.

20. Nakagawa-Yagi Y, Hara H, Nakagawa F, Sato M, Hara A (2016) Acute exposure to an electric field induces changes in human plasma 9-HODE, 13-HODE, and immunoreactive substance P levels: Insight into the molecular mechanisms of electric field therapy. Integr Mol Med 3: 600-605.

21. Nakagawa-Yagi Y, Hara H, Tsuboi H, Abe J, Hara A (2016) Effect of 3-hydroxybutyrate, an endogenous histone deacetylase inhibitor, on $F O X O 3 \mathrm{~A}$ mRNA expression in human epithelial colorectal Caco-2 cells: Insight into the epigenetic mechanisms of electric field therapy. Integr Mol Med 3: 764-768.

22. Taguchi R, Houjou T, Nakanishi H, Yamazaki T, Ishida M, et al. (2005) Focused lipidomics by tandem mass spectrometry. $J$ Chromotogr B Analyt Technol Biomed Life Sci 823: 26-36.

23. Nakanishi H, Iida Y, Shimizu, Taniguchi R (2009) Analysis of oxidized phosphatidylcholines as markers for oxidative stress, using multiple reaction monitoring with theroretically expanded data sets with reversed-phase liquid chromatography/ tandem mass spectrometry. J Chromotogr B Analyt Technol Biomed Life Sci 877: 13661374.

24. Murphy RC, Gaskell SJ (2011) New applications of mass spectrometry in lipid analysis. J Biol Chem 286: 25427-25433.

25. Hinterwirth H, Stegemann C, Mayr M (2014) Lipidomics: Quest for molecular lipid biomarkers in cardiovascular disease. Circ Cardiovasc Genetics 7: 941-954.

26. Yang K, Han X (2016) Lipidomics: Techniques, applications, and outcomes related to biomedical sciences. Trends Biochem Sci 41: 954-969.

27. Mihara H, Boudaka A, Shibasaki K, Yamanaka A, Sugiyama T, et al. (2010) Involvement of TRPV2 activation in intestinal movement through nitric oxide production in mice. $J$ Neurosci30: 16536-16544.

28. Imae R, Inoue T, Nakasaki Y, Uchida Y, Ohba Y, et al. (2012) LYCAT, a homologue of C. elegans acl-8, acl-9, and acl-10, determines the fatty acid composition of phosphatidylinositol in mice. J Lipid Res 53: 335-347.

29. Baba T, Kashiwagi Y, Arimitsu N, Kogure T, Endo A, et al. (2014) Phosphatidic acid (PA)-preferring phospholipase A1 regulates mitochondrial dynamics. J Biol Chem 289: 11497-11511.

30. Bligh EG, Dyer WJ (1959) A rapid method of total lipid extraction and purification Canadian J Biochem Physiol 37: 911-917.

31. Kielkowska A, Niewczas I, Anderson KE, Durrant TN, Clark J, et al. (2014) A new approach to measuring phosphoinositides in cells by mass spectrometry. Adv Biol Regul 54: 131-141.

32. Trott O, Olson AJ (2010) AutoDockVina: Improving the speed and accuracy of docking with a new scoring function, efficient optimization, and multithreading. $J$ Comput Chem 31: 455-461.

33. Berthold MR, Cebron N, Dill F, Gabriel TR, Kotter T, et al. (2007) KNIME: The Konstanz Information Miner. Springer, InStudies in Classification, Data Analysis, and Knowledge Organization 319-326.

34. Thuren T, Tulkki AP, Virtanen JA, Kinnunen PK (1987) Triggering of the activity of phospholipase A2 by an electric field. Biochemistry 26: 4907-4910.

35. Balsinde J, Balboa MA (2005) Cellular regulation and proposed biological functions of group VIA calcium-independent phospholipase A2 in activated cells. Cell signal 17: $1052-1062$.

36. Nakagawa-Yagi Y, Sato Y, Matsumoto E, Nakatsuka S, Sakaki T, et al. (2012) Pharmacological modulation of histone demethylase activity by a small molecule isolated from subcritical water extracts of Sasasenanensis leaves prolongs the lifespan of Drosophila melanogaster. BMC Complement Altern Med 12: 101.

37. Azam SS, Abbasi SW (2013) Molecular docking studies for the identification of novel melatoninergic inhibitors for acetylserotonin-O-methyltransferase using different docking routines. Theoretical Biol Medical Modelling 10: 63.

38. Huynh KW, Cphen MR, Jiang J, Samanta A, Lodowski DT, et al. (2016) Structure of the full-length TRPV2 channel by cryo-EM. Nat Commun 7: 11130.

39. Zubcevic L, Herzik MA, Chung BC, Liu Z, Lander GC, et al. (2016) Cryo-electron microscopy sturucture of the TRPV2 ion channel. Nat Stuct Mol Biol 23: 180-186.

40. Szolcsanyi J, Sandor Z (2012) Multisteric TRPV1 nocisensor: a target for analgesics. Trends Pharmacol Sci 33: 646-655.

41. Lazaro MSL, Simon SA, Rosenbaum T (2013) The role of endogenous molecules in modulating pain through transient receptor potential vanilloid 1 (TRPV1). $J$ Physiol591: 3109-3121.

42. Julius D (2013) TRP channels and pain. Annu Rev Cell Dev Biol 29: 355-384.

43. Nagy I, Friston D, Valente JS, Perez JVT, Andreou AP, et al. (2014) Pharmacology of the capsaicin receptor, transient receptor potential vanilloid type-1 ion channel. In: Abdel-Salam OME (ed.), Capsaicin as a Therapeutic Molecule. Progress in Drug Research 68: 39-76.

44. Prescott ED, Julius D (2003) A modular PIP 2 binding site as a determinant of capsaicin receptor sensitivity. Science 300: 1284-1288.

45. Posadas NA, Juarez PG, LIorente I, OsegueraJA, Lazaro MS, et al. (2012) Lysophosphatidic acid directly activates TRPV1 through a C-terminal binding site. Nat Chem Biol 8: 78-85.

46. Marin PA, MacianDP, Gaudet R (2013) What do we know about the transient receptor 
Nakagawa-Yagi Y (2017) Acute exposure to an electric field induces changes in human plasma lysophosphatidylcholine (lysoPC)-22:4 levels: Molecular insight into the docking of lysoPC-22:4 interaction with TRPV2

potential vanilloid 2 (TRPV2) ion channel? FEBSJ 280: 5471-5487.

47. Zhang F, Hanson SM, Jara-Oseguera A, Krepkiy D, Bae C, et al. (2016)Engineering vanilloid-sensitivity into the rat TRPV2 channel. ELIFE 5: e16409.

48. Andersson DA, Nash M, Bevan S (2007) Modulation of the cold-activated channel TRPM8 by lysophospholipids and polyunsaturated fatty acids. J Neurosci 27: 3347 3355.
49. Lauffer LM, Iakoubov R, Brubaker PL (2009) GPR119 is essential for oleoeylethanolamide-induced glucagon-like peptide-1 secretion from the intestinal enteroendocrine L-cell. Diabetes 58: 1058-1066.

50. Hansen HS, Rosenkilde MM, Holst JJ, Schwartz TW (2012) GPR119 as a fat sensor. Trends Pharmacol Sci 33: 374-381.

Copyright: (C2017 Nakagawa-Yagi Y. This is an open-access article distributed under the terms of the Creative Commons Attribution License, which permits unrestricted use, distribution, and reproduction in any medium, provided the original author and source are credited. 